\title{
L'Attention
}

\section{La dynamique pré-réfléchie de l'expérience vécue}

\section{Claire Petitmengin}

\section{(2) OpenEdition}

Journals

Édition électronique

URL : http://journals.openedition.org/alter/1668

DOI : $10.4000 /$ alter.1668

ISSN : 2558-7927

\section{Éditeur :}

Association ALTER, Archives Husserl (CNRS-UMR 8547)

\section{Édition imprimée}

Date de publication : 1 octobre 2010

Pagination : 165-182

ISBN : 2-9522374-6-8

ISSN : $1249-8947$

\section{Référence électronique}

Claire Petitmengin, «La dynamique pré-réfléchie de l'expérience vécue », Alter [En ligne], 18 | 2010, mis en ligne le 01 juin 2020, consulté le 28 juin 2020. URL : http://journals.openedition.org/alter/1668 ; DOI : https://doi.org/10.4000/alter.1668 


\section{LA DYNAMIQUE PRÉ-RÉFLÉCHIE DE L'EXPÉRIENCE VÉCUE}

Claire Petitmengin

\section{Introduction}

Ce texte explore une dimension profondément «pré-réfléchie » de notre expérience vécue, qui semble jouer un rôle essentiel dans la genèse d'une perception, mais aussi d'une compréhension, d'un sens. A partir de plusieurs exemples, et notamment celui de l'écoute d'un son, je montrerai que cette dimension possède des caractéristiques structurelles spécifiques, qui semblent très différentes de celles de l'expérience dont nous sommes habituellement conscients. Notamment, la distinction entre un espace " intérieur » et un espace " extérieur », entre un sujet percevant et un objet perçu, et entre les différentes modalités sensorielles, y est beaucoup plus perméable. Je m'intéresserai également aux micro-opérations pré-réfléchies que nous réalisons pour entrer en contact avec cette dimension, ainsi qu'à la mise en disposition attentionnelle qui permet d'en acquérir une conscience réfléchie. Ces investigations $\mathrm{m}^{\prime}$ amèneront à $\mathrm{m}$ 'interroger sur la pertinence du terme « pré-réfléchi » lui-même.

\section{Définition du pré-réfléchi}

Par expérience «pré-réfléchie », j'entends la part de notre expérience qui est vécue sans être reconnue, sans être immédiatement accessible à la conscience et à la description verbale. C'est la part de notre expérience qui est ordinairement oblitérée par l'absorption de notre attention dans le contenu, l'objet de notre activité. Par exemple, lorsque je fais un mouvement, mon intérêt pour l'objet vers lequel le mouvement est dirigé - le ballon, la pomme - occulte le mouvement de mon bras, qui lui-même occulte les sensations internes que j'éprouve dans les muscles et les articulations qui 


\section{L'attention}

initient en fait le mouvement de mon bras ${ }^{1}$. Lorsque je lis un roman, l'absorption de mon attention dans l'histoire, occulte la conscience de mon activité de lecture - par exemple les mouvements de mes yeux, les mots prononcés silencieusement, la succession rapide d'images internes, les émotions légères qui accompagnent cette activité. Si je regarde un paysage, ou si je contemple un tableau, j'y reconnais instantanément des éléments dans lesquels mon attention s'absorbe. Mon attention se focalise sur les objets, là-bas, je perds le contact mon expérience immédiate. Qu'il s'agisse de percevoir, de mémoriser, de se souvenir, mais aussi d'imaginer, de calculer, de comprendre ou de décider... l'absorption dans l'objet ou l'objectif, le «quoi » du processus, occulte le «comment », qui reste ordinairement non remarqué, non reconnu ${ }^{2}$.

Curieusement, nous ne sommes pas conscients de cette déficience de conscience. Nous n'imaginons pas qu'un travail particulier soit nécessaire pour prendre conscience de ce qui nous est le plus proche, le plus intime. Ce qui est le premier et le principal obstacle à la prise de conscience : pourquoi, en tant qu'individu, me mettrais-je en quête d'acquérir une conscience dont je ne sais pas qu'elle me manque ${ }^{3}$ ? Pourquoi, en tant que chercheur, me donnerais-je le projet de concevoir des méthodes permettant de développer une telle conscience?

Pourtant je peux, moyennant un certain entraînement, ou avec l'assistance d'une personne experte, apprendre à réorienter mon attention vers cette part pré-réfléchie et la reconnaître.

\section{Méthodes d'accès au pré-réfléchi}

Personnellement, c'est la pratique de plusieurs méthodes empiriques destinées à susciter un aiguisement et une réorientation de l'attention, qui m'ont amenée à découvrir l'importance de cette dimension, et à l'explorer. La première est l'entretien d'explicitation, méthode conçue par Pierre Vermersch pour susciter chez autrui les différents gestes intérieurs qui

1. R. Shusterman, Body Consciousness. A Philosophy of Mindfulness and Somaesthetics, Cambridge University Press, 2008, p. 94.

2. Ce mouvement "naturel », «spontané » en direction de l'objet au détriment de l'expérience que j'en ai a généralement été décrit par Husserl comme relevant de «l'attitude naturelle » (Idées directrices pour une philosophie et une phénoménologie philosophique pure I, Paris, Gallimard, $1913, \S 27)$. E. Fink parle quant à lui de Weltbefangenheit (enfermement dans le monde) dans la Sixième Méditation cartésienne (Grenoble, J. Millon, 1994, §5). Le mouvement inverse de conversion réflexive est très précisément décrit pas Husserl comme un passage du «quoi » au « comment» (par exemple dans Méditations cartésiennes, Paris, PUF, 1994, §2). Pourtant, ce mouvement, tout en étant nommé en des termes très proches voire identiques par les phénoménologues, n'est pas décrit dans sa dimension procédurale, pratique et concrète en termes d'activité incarnée, corporelle et gestuelle, comme je vais tenter de le faire ici. Pour une amorce de cette orientation procédurale, cf. On Becoming Aware. A Pragmatics of Experiencing, Amsterdam, Benjamins Press, 2003, section I, « Épochè »).

3. P. Vermersch, «Conscience directe et conscience réfléchie », Intellectica 2000/2: 31, p. 269-311. 
permettent de passer d'une conscience pré-réfléchie à une conscience réfléchie de son expérience, et de décrire cette expérience avec une grande précision ${ }^{4}$. La deuxième est une méthode de méditation issue du bouddhisme indien, nommée en sanskrit shamatha-vipashyana ${ }^{5}$. Shamatha ${ }^{6}$, la pratique du «calme mental », permet d'apprendre à stabiliser son attention et à apaiser le flot des phénomènes mentaux, grâce à la concentration de l'esprit sur un unique objet. Vipashyana ${ }^{7}$, la pratique de la "vision pénétrante ", consiste à appliquer l'attention aiguisée par shamatha au flux de l'expérience ainsi apaisé, afin de d'apprendre à reconnaître les phénomènes qui émergent, d'instant en instant, et à prendre conscience de la structure dynamique de l'expérience. Une troisième méthode, la méthode du focusing conçue par le philosophe et psychothérapeute américain Eugene Gendlin ${ }^{8}$, est tout particulièrement adaptée à la prise de conscience de sensations corporelles subtiles.

La pratique de ces méthodes m'a amenée à découvrir, non sans surprise, l'importance de la part pré-réfléchie de ma propre expérience, et chez un grand nombre d'autres personnes par le biais d'entretiens d'explicitation.

L'émergence à la conscience d'un élément de l'expérience initialement pré-réfléchi suscite en effet de la surprise. J'ai l'impression de reconnaître quelque chose de très familier, avec lequel je vivais depuis toujours, mais qui restait non remarqué. Cette reconnaissance s'accompagne d'un sentiment d'évidence, d'apodicticité. "C'est conforme à ce que je suis sûr d'avoir vécu - dit un sujet interviewé - mais que je n'avais pas conscience de vivre au moment où je l'ai vécu. »

Je vais présenter ici certains résultats de ce travail empirique, en signalant seulement quelques pistes de rapprochement avec la tradition phénoménologique, une comparaison approfondie n'étant pas le propos de cet article.

\section{Les « strates » d'expérience pré-réfléchie}

Ces différentes pratiques montrent que l'expérience pré-réfléchie semble posséder différentes "strates", dont l'accès à la conscience réfléchie est de plus en plus difficile.

Par exemple, dans la pratique de la méditation shamatha-vipashyanâ, le méditant commence souvent par découvrir, non sans étonnement, l'importance

4. P. Vermersch, L'entretien d'explicitation, Paris, ESF, 1994/2008

5. Par exemple, C. Genoud, "On the cultivation of presence in Buddhist meditation ", Journal of Consciousness Studies 16 (2009), 11-12, p. 117-128.

6. Dans le terme shama-tha, shama signifie «paix» et tha « reposer, demeurer».

7. Dans le terme vi-pashyanâ, pashyanâ signifie «vision, regard », et vi marque la discrimination et l'intensité.

8. E. Gendlin, Experiencing and the Creation of Meaning, Northwestern University Press, 1962/1997 et Focusing : au centre de soi, Québec, Le Jour éditeur, 1994. 


\section{L'attention}

de son discours intérieur, qu'il s'agisse de monologues ou de dialogues imaginaires. D'ailleurs il arrive fréquemment qu'un méditant débutant ait l'impression que la pratique de la méditation intensifie ce discours, alors qu'il prend seulement conscience de son omniprésence. Le méditant découvre aussi, accompagnant cette rumeur presque ininterrompue, un flot rapide d'images et de "films » intérieurs : souvenirs proches ou lointains, agréables ou non, scènes futures désirées ou appréhendées, dont seule une petite part apparaît à la conscience. Ces discours imaginaires et cette imagerie intérieure contribuent à entretenir un flux presque incessant d'émotions, dont seules les plus intenses sont ordinairement perçues. Mais ces strates discursive, imaginaire et émotionnelle occultent une dimension encore plus difficile d'accès, plus subtile.

\section{L'expérience d'écoute}

Pour illustrer les étapes de ce processus de descente vers des strates de plus en plus profondément pré-réfléchies, prenons l'exemple de l'expérience associée à l'écoute d'un son. Je résumerai pour cela les résultats d'une étude réalisée dans le cadre du CREA $^{9}$ par une équipe de recherche pluridisciplinaire nommée "groupe de recherche neuro-expérientiel ». Pendant un an, nous avons consacré du temps à écouter différents types de sons, à expliciter ces expériences, et à analyser les descriptions produites. Ce travail nous a permis de déceler une structure générique à trois dimensions de l'expérience d'écoute, selon que l'attention du sujet se porte 1) sur l'événement qui est à la source du son, 2) sur le son en tant que tel, considéré indépendamment de sa source, 3) ou sur le ressenti corporel du son. Ce travail nous a amenés à faire l'hypothèse que ces trois dimensions correspondent à des aspects de plus en plus profondément pré-réfléchis de l'expérience, qui s'occultent l'un l'autre ${ }^{10}$.

\section{Objet source du son}

Un son se produit. Si l'on me demande de décrire ce qui s'est passé, ce que je décris ordinairement immédiatement, c'est l'événement physique qui est à la source du son : "Quelqu'un a cassé un verre dans la cuisine », "C'est le bruit du vent dans les arbres", "C'est Jean qui scie du bois dans le jardin ». Comme l'écrit Don Ihde, "les sons sont d'abord "expériencés » (experienced) comme les sons de choses ${ }^{11} »$. Le son me renseigne sur les

9. Centre de recherche en épistémologie appliquée (École polytechnique/CNRS), en collaboration avec le Réseau «Philosophie et neurosciences " (Institut de neurosciences cognitives de la méditerranée-CNRS).

10. C. Petitmengin, M. Bitbol, J.M. Nissou, B. Pachoud, H. Curalucci, M. Cermolacce, J. VionDury, « Listening from Within », Journal of Consciousness Studies 16 (2009), 10-12, p. 252-284.

11. D. Ihde, Listening and Voice. Phenomenologies of Sound, State University of New York Press, 2007 (1re édition Ohio University Press, 1976), p. 60. 
caractéristiques du ou des objets qui ont produit ce son : leur direction, leur distance, leur vitesse, leur densité, leur caractère plein ou creux, la consistance de leur surface. L'attention ne se porte sur le son lui-même que dans la mesure où il permet d'identifier la source du son, dont l'image vient rapidement occulter le son entendu. Un son se produit, et en une fraction de seconde, je reconnais ce phénomène comme le chant d'un merle qui arrive par la fenêtre de mon bureau, sans plus m'intéresser aux particularités de ce chant.

Cette focalisation de l'attention sur l'objet qui est à la source du son a un impact sur la structure de l'espace vécu. Le sujet «se quitte » en quelque sorte pour se prolonger vers la source du son, «oubliant » en quelque sorte les sensations corporelles liées à cette expérience, dont il ne garde qu'une conscience pré-réfléchie. Il est un peu comme l'aveugle qui explore un objet du bout de sa canne, dont l'attention est entièrement absorbée par l'objet exploré, et qui n'a qu'une conscience pré-réfléchie du contact de la canne dans la paume de sa main, pour reprendre un exemple célèbre. Le son, le milieu sonore et le corps lui-même deviennent comme transparents.

\section{Son entendu}

Un son se produit. Je peux aussi retourner mon attention vers ce son en tant que son, indépendamment de sa source ${ }^{12}$. Le musicologue français Pierre Schaeffer qualifie $\mathrm{d}^{\prime}$ " acousmatique ${ }^{13}$ » ce mode d'écoute qui se focalise sur les qualités intrinsèques du son en tant qu'objet purement auditif ${ }^{14}$. Le son n'est plus considéré comme un indice, un medium me renseignant sur autre chose, mais il est perçu immédiatement pour lui-même. Par exemple, j'écoute le chant du merle en tant que chant, oubliant même qu'il s'agit du chant d'un merle. Un peu comme l'aveugle qui détournerait son attention de l'objet exploré vers les caractéristiques tactiles de la canne dans la paume de sa main : lisse, rond, froid. Ce retournement de l'attention vers les qualités du son me permet d'en acquérir une conscience réfléchie, et de discerner des nuances que l'absorption de l'attention dans la source oblitère habituellement. Ce mode d'attention est moins directionnel, plus ouvert, plus diffus que celui qui est focalisé sur la source. Le chant du merle arrive

12. Ce geste n'est autre que la «conversion phénoménologique » husserlienne, qui consiste à détourner l'attention des objets qui apparaissent à la conscience, pour l'orienter vers les modes subjectifs d'apparition de ces objets: nous devons "détourner notre regard des choses, des pensées, des valeurs correspondantes, qu'il prend pour thème, et l'orienter vers la multiplicité changeante des "modes subjectifs » qui les font apparaître tels que nous en avons conscience. » (E. Husserl, «La Phénoménologie », article pour l'Encyclopedia Britannica traduit de l'allemand par J.-F. Courtine et J.-L. Fidel dans Notes sur Heidegger, Paris, Éditions de Minuit, 1993, p. 75).

13. Le terme provient du grec ancien akousmatikoi, nom donné aux disciples de Pythagoras qui écoutaient leur maître à travers un rideau. Le corps physique de Protagoras leur était caché, seul le son de sa voix les atteignait.

14. P. Schaeffer, Traité des objets musicaux, Paris, Seuil, 1966. 


\section{L'attention}

du jardin. Mais si je change de mode d'attention et écoute ce chant comme j'écouterais de la musique, cette directionnalité s'estompe. Le chant remplit l'espace. Le milieu, devenu comme transparent dans le mode d'écoute précédent, prend ici une certaine densité, une certaine épaisseur.

\section{Son ressenti}

Un son se produit. Je peux aussi détourner mon attention de la source du son (de la question «quel est ce son ?»), et du son en tant que son (de la question «comment est ce son ?»), pour la tourner vers... « ce que cela me fait d'écouter ce $\operatorname{son}^{15} »$. Pour prendre une analogie visuelle, je ne $\mathrm{m}^{\prime}$ intéresse plus au vase bleu, ni au bleu du vase, mais je m'intéresse au ressenti du bleu, à ce que cela me fait de regarder ce bleu.

Le son résonne dans notre corps. Cette résonnance est parfois très aisément perceptible, comme celle des basses dans un concert de rock ou une boîte de nuit, ou celle d'un marteau-piqueur. Mais il est des résonnances plus subtiles. Par exemple avez-vous déjà fait l'expérience de ressentir physiquement l'arrivée d'un avion ou d'un bateau plusieurs minutes avant que le son n'en soit audible ? Un certain entraînement, l'adoption d'une disposition attentionnelle particulière, permet de prendre conscience de ressentis encore plus subtils, comme celui que suscite la voix d'autrui, le chant d'un oiseau ou même le bruissement du feuillage. Au lieu d'aller chercher le son, de "tendre l'oreille» vers lui pour le caractériser, cette disposition consiste à $\mathrm{s}^{\prime} \mathrm{y}$ rendre réceptif, à laisser le son venir à soi, à se laisser «toucher» par le son ${ }^{16}$. Un peu comme l'aveugle qui cesserait d'explorer activement les caractéristiques tactiles de la canne, pour tourner son attention vers les sensations internes ressenties dans la paume de sa main, qui au lieu de toucher la canne se laisserait toucher par elle ${ }^{17}$.

À quoi ressemble le son ressenti ? Il nous a été décrit comme un battement, ou une caresse, parfois comme une pulsation, parfois comme un frémissement... Le son ressenti est fait de modifications dynamiques

15. Comme l'écrit G.E. Moore, après avoir soutenu que la sensation du bleu n'est rien d'autre que le bleu, et qu'elle s'évanouit si nous essayons de fixer notre attention sur elle : "Yet it can be distinguished if we look enough, and if we know that there is something to look for » (G.E. Moore, «The refutation of idealism », Mind 12, 1903, p. 450)

16. De nombreuses descriptions de cette disposition attentionnelle propice à la prise de conscience de la dimension pré-réfléchie de l'expérience, et des "gestes intérieurs" qui permettent de l'adopter, ont été recueillies dans C. Petitmengin, L'expérience intuitive, Paris, L'Harmattan, 2001 (pp. 183-191 and 251-268). Cette disposition est très voisine de celle que N. Depraz, F. Varela, et P. Vermersch nomment letting go (lâcher prise) dans On Becoming Aware. A Pragmatics of Experiencing (Amsterdam, Benjamins Press, 2003, chapitre 1.2). Toutefois, le terme letting come (laisser advenir) me paraît mieux adapté pour caractériser cette attitude de réceptivité et d'accueil, et je préfèrons réserver le terme « lâcher prise » au geste qui y prépare. 17. Pour reprendre le célèbre exemple de Merleau-Ponty de la main qui peut être touchante ou touchée (par exemple Phénoménologie de la perception, p. 108-109, Le visible et l'invisible, p. 164-165). 
insaisissables d'intensité et de rythme. Dans la musique, ce ressenti correspond à c'est ce qui n'est pas codé par des notes, mais par des notations dynamiques comme " crescendo», "staccato», «piano», "forte»... Ces rythmes ou pulsations subtiles sont particulièrement appréhendables dans l'expérience musicale, mais une attention fine permet aussi d'en prendre conscience dans l'expérience que suscitent en nous une voix, un bruit. En fonction de la hauteur et de l'intensité du son, ces rythmes sont ressentis au niveau de la tête, de la poitrine, du ventre, plus ou moins profondément, parfois dans le corps tout entier. "Je suis, dit un sujet, rempli de cette matière sonore, comme si j'étais devenu un violon ou une cloche sur laquelle on jouerait. ${ }^{18}$ » Dans ce mode d'écoute, le son semble perdre son identité de son. Ce mode d'écoute a lui aussi un impact sur la structure de l'espace vécu : une sorte de synchronisation, d'accordage rythmique se produit entre l'espace corporel et l'espace perçu comme « extérieur », qui a pour effet de rendre plus beaucoup plus perméable la limite entre ces deux espaces. "C'est comme si l'extérieur devenait plus dense - dit un sujet - et l'intérieur moins compact, plus vibrant, et progressivement la texture des deux s'harmonise. Cette vibration abolit les limites de mon corps.»C'est précisément ce type d'expérience que décrit le poète Rilke en évoquant l'instant «où il y eut, au dehors et au-dedans de lui, et accordant l'un à l'autre, un cri d'oiseau qui, en quelque sorte, ne se brisait pas à la frontière du corps et réunissait les deux côtés en un seul espace ininterrompu ${ }^{19}$ ».

$\mathrm{Au}$ cours du processus de conversion de l'attention de la source du son vers le son entendu, puis du son entendu vers le son ressenti, l'effort réalisé pour saisir et caractériser un objet se relâche donc progressivement pour laisser la place à une attitude de réceptivité, d'accueil. Cette perte d'intentionnalité semble s'accompagner d'une synchronisation progressive entre l'espace perçu comme «intérieur» et l'espace perçu comme "extérieur", qui a pour effet d'affaiblir (d'attendrir en quelque sorte) la distinction entre les deux. Autrement dit, plus l'attention se détache de son absorption dans les objets extérieurs pour entrer en contact avec l'expérience dite «intérieure », plus la distinction entre « intérieur » et « extérieur », soi et non soi, se fragilise ${ }^{20}$.

18. H. Werner, «L'unité des sens», Journal de psychologie normale et pathologique, 31 (1934), 190205 (p. 199).

19. R.M. Rilke, «Instant vécu», dans Lettres à un jeune poète, suivi de Proses, Paris, Librairie Générale Française (Le Livre de Poche), 1989, p. 118.

20. C'est la raison pour laquelle le geste de redirection de l'attention de «l'extérieur» vers «l'intérieur» décrit dans On becoming aware comme constitutif du processus de prise de conscience ne semble à mon sens concerner que les phases initiales du processus: plus la dimension explorée devient profondément pré-réfléchie, plus la séparation ordinairement perçue entre intérieur et extérieur devient perméable, plus le geste de redirection tend à s'atténuer, jusqu'à disparaître. 


\section{L'attention}

\section{Autres aperçus de la dimension ressentie}

Il semble que les trois positions attentionnelles que nous avons identifiées dans l'expérience auditive se retrouvent dans les autres modalités sensorielles. Ainsi, lorsque je contemple un paysage, je peux $\mathrm{m}^{\prime}$ attacher à identifier les objets qui le composent: ruisseau, peupliers, bouleaux. Un mode $\mathrm{d}$ 'attention moins directionnel m'amène à oublier les objets pour m'intéresser par exemple aux contrastes d'ombres et de lumières, ou aux couleurs du paysage, ce qui me permet de distinguer des nuances de vert, ou des reflets roses dans l'eau, que la reconnaissance immédiate d'objets occulte habituellement. En adoptant un mode attentionnel encore plus ouvert et réceptif, je peux aussi laisser la couleur venir à moi, m'imprégner, un peu comme le ferait un parfum.

\footnotetext{
"Tout d'un coup - dit un sujet - j'ai ressenti effectivement ce que c'était que regarder. Regarder, ce n'est pas lancer ton regard vers quelque chose, le projeter, le tendre, mais laisser la couleur, ou le paysage, venir à toi. Tu ne vas pas chercher, tu recueilles, tu reçois. Et tu as l'impression qu'effectivement, la couleur ou le paysage s'imprègne à l'intérieur de toi. »
}

Lorsque nous commençons à reconnaître ces ressentis subtils, et à savoir adopter la disposition attentionnelle qui nous permet d'en acquérir une conscience réfléchie, nous les repérons de plus en plus rapidement dans tous les domaines de notre expérience. Par exemple, dans l'expérience de la rencontre avec une œuvre d'art. Imaginez que vous vous trouvez devant un tableau que vous aimez particulièrement, par exemple dans un musée. Prenez quelques instants pour porter votre attention sur le ressenti que suscite en vous ce tableau. C'est un sentiment vague, diffus, difficilement descriptible mais néanmoins intense et spécifique (il serait très différent si vous vous imaginiez en présence d'un autre tableau). La même expérience pourrait être réalisée en évoquant par exemple une poésie, ou même un roman, dont le titre seul va susciter tout un monde complexe d'impressions fugitives, indistinctes, mais riches de sens.

Nous retrouvons la même dimension dans la phase initiale de l'émergence de certains souvenirs. Parfois, le souvenir surgit d'un bon, à l'improviste, sous une forme sensorielle d'emblée précise. Mais d'autres fois, il est lent à émerger, tarde à prendre une forme précise: l'étape ordinairement infinitésimale de son émergence se stabilise pendant quelques instants, parfois même quelques heures, ce qui nous laisse le temps de porter notre attention sur cette expérience étrange, si subtilement décrite par Proust dans $D u$ côté de chez Swann. Avant même que nous puissions reconnaître la situation dont il s'agit et la nommer, avant même que ne surgissent des images, des sons, et des émotions précises et identifiables, s'impose à nous 
un ressenti non sensoriellement différencié, mais néanmoins spécifique et intense, chargé d'une densité charnelle et vivante ${ }^{21}$.

Ou encore, en présence d'une autre personne, la perception visuelle que nous avons des expressions de son visage, de ses mouvements, l'échange verbal qui s'instaure, la tonalité émotionnelle qu'elle suscite en nous, etc. accaparent notre attention, voilant l'impression plus diffuse, globale et complexe, tout à fait spécifique (très différente d'une personne à l'autre), éprouvée en sa présence. Impression qui est plus accessible lors d'une toute première rencontre, ou après une longue séparation.

Comme beaucoup de psychothérapeutes l'ont remarqué, c'est à ce niveau-là que se joue une thérapie. Car un patient peut comprendre son problème de manière conceptuelle en quelques heures, et être capable de l'expliquer, sans pour autant en être libéré. Cette libération intervient au cours d'un processus expérientiel généralement plus long, qui consiste à prendre conscience du «sens ressenti» (felt meaning) de son problème comme dit Gendlin - pour l'amener progressivement à se transformer ${ }^{22}$. Le ressenti du problème va peu à peu se détendre, s'élargir, se diluer. C'est un processus de compréhension et de transformation quasi-corporelle, qui ressemble beaucoup plus à un travail de distillation intérieure, qu'à un arrangement de concepts. L'expression verbale va jouer un rôle très important dans ce processus, mais seulement dans la mesure où elle contribue à cette alchimie, à cette transformation profonde de la matière concrète de notre expérience.

Cette dimension « ressentie » semble même jouer un rôle essentiel dans la pensée abstraite. Par exemple, la solution d'un problème personnel, l'idée nouvelle... apparaît parfois d'un seul coup, complète, sous la forme d'un insight instantané. Mais bien souvent, avant de prendre une forme précise et communicable, l'idée affleure d'abord à la conscience comme un ressenti vague et flou, une direction de pensée, une ligne de force qui guide silencieusement la recherche, comme l'avait observé Einstein :

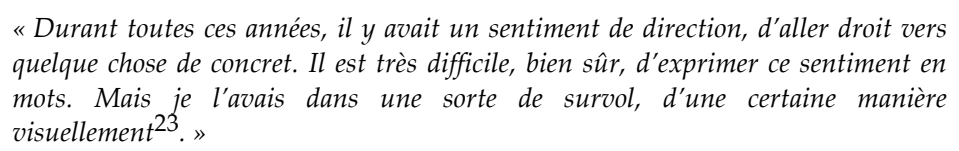

"Durant toutes ces années, il y avait un sentiment de direction, d'aller droit vers quelque chose de concret. Il est très difficile, bien sûr, d'exprimer ce sentiment en mots. Mais je l'avais dans une sorte de survol, d'une certaine manière visuellement ${ }^{23}$. »

Ce «sens ressenti » de l'idée se déploie et se précise au cours du temps, au rythme des rencontres, des discussions, des lectures faites par le

21. G. Gusdorf, Mémoire et personne, Paris, Presses universitaires de France, 1950.

22. E. Gendlin, Focusing-Oriented Psychotherapy. A Manual of the Experiential Method, New York, The Guilford Press, 1996

23. Réponse d'Einstein au psychologue Max Wertheimer qui l'interrogeait « en grand détail sur les événements concrets dans ses pensées l'ayant conduit à la théorie de la relativité » (cité par Holton, L'invention scientifique : themata et interprétation, Paris, Presses Universitaires de France, 1982, p. 440-441). 


\section{L'attention}

chercheur... il est la matière vivante (bien qu'ordinairement pré-réfléchie) de son travail. Et même lorsque l'idée a été explicitée et formalisée, il continue à vivre de manière souterraine, c'est en lui que l'idée trouve sens et chair.

\section{Caractéristiques structurelles de la dimension ressentie}

Quelles sont les caractéristiques structurelles de cette dimension « ressentie ${ }^{24} » ?$

\section{Dimension ressentie et « marge»}

Remarquons tout d'abord que la dimension ressentie ne doit pas être confondue avec «l'arrière-plan» («l'horizon», la «marge») d'une perception : lorsque nous focalisons notre attention sur un objet donné, nous percevons en même temps de manière floue le « fond » - formes indistinctes, couleurs - sur lequel il se détache. Il suffit généralement que nous dirigions notre attention sur cet arrière-plan, pour le percevoir à son tour distinctement, entouré lui-même de son propre arrière-plan. Mais la dimension ressentie, même lorsqu' on dirige son attention dessus, reste floue et indistincte.

\section{Spécificité}

Bien que vague et flou, un sens ressenti est généralement spécifique, déterminé, il ne peut pas être confondu avec un autre. Le sens ressenti que j'ai d'une personne est tout à fait différent de celui que j'ai d'une autre. Et le sens ressenti que j'éprouve lorsque je cherche les mots pour exprimer une idée est tout à fait différent d'une idée à l'autre.

\section{Transmodalité}

Dans la dimension ressentie, la frontière entre les différentes modalités sensorielles est beaucoup plus perméable que dans notre expérience plus consciente. L'analyse des descriptions recueillies montre que le vocabulaire utilisé pour décrire la « texture » de la dimension ressentie fait souvent appel simultanément à plusieurs registres sensoriels. Par exemple, le «sentiment de direction » qui guide Einstein dans ses recherches semble être à la fois d'ordre kinesthésique et visuel. Ou pour prendre un exemple dans un tout autre domaine, le psychanalyste Theodor Reik décrit les sensations internes subtiles qui le guident au cours d'une séance tantôt comme visuelles («nuances et ombres psychiques fuyantes»), tantôt comme auditives

24. Cette section s'inspire de C. Petitmengin, «Towards the source of thoughts. The gestural and transmodal dimension of lived experience ", Journal of Consciousness Studies, vol. 14, $\mathrm{n}^{\circ} 3$ (2007), p. 54-82. 
(« demi-tons quasi imperceptibles $\left.{ }^{25} »\right)$, tantôt comme tactiles («petites incohérences, légères irrégularités qui ne sont pas visibles mais perceptibles au toucher comme lorsque les mains glissent soigneusement et doucement sur le tissu $26 »)$.

$\mathrm{Si}$ la dimension ressentie ne relève pas d'une modalité sensorielle déterminée, elle a en réalité des submodalités sensorielles précises. Ces submodalités, qui sont l'intensité, le rythme et le mouvement, ont pour caractéristique d'être «transmodales», c'est-à-dire qu'elles ne sont particulières à aucun sens mais transposables d'un sens à un autre (par opposition par exemple à la température et à la texture qui sont spécifiques au toucher, à la couleur qui est spécifique à la vue...). Ce sont les « sensibles communs » qu'avaient déjà repérés Platon ${ }^{27}$ et Aristote $^{28}$.

De manière intéressante, il semble que cette dimension transmodale corresponde au monde que le petit enfant expérimente. C'est ce que Daniel Stern a mis en évidence à partir d'observations très précises des interactions entre mère et enfant, complétées par des "entretiens micro-analytiques » avec les mères ${ }^{29}$. Le monde que le nourrisson expérimente - écrit Stern n'est pas un monde d'images, de sons et de sensations tactiles, mais de modifications dynamiques subtiles de mouvements, d'intensités et de rythmes, c'est-à-dire de qualités transmodales, transposables d'une modalité à l'autre, qu'il appelle « dynamiques de vitalité » (vitality dynamics).

\section{Caractère gestuel et rythmique}

Le nourrisson en effet ne perçoit pas les actes comme tels, comme le font les adultes (atteindre le biberon, déplier la couche), mais les dynamiques de vitalité liées à ces actes (comment sa mère le prend, prend le biberon, plie les couches, se peigne ou peigne son bébé...). Ce sont ces rythmes qui permettent l'accordage affectif (affect attunement dit Stern) entre la mère et l'enfant. D'instant en instant, de manière pré-réfléchie, mère et enfant accordent leur rythme interne: par exemple, la mère va répondre à un babillement du bébé par une caresse de même intensité et de même rythme. Cette synchronisation rythmique, qui permet la résonance, l'accord entre deux univers intérieurs, semble être la base de l'intersubjectivité affective.

Les travaux de Stern l'amènent en effet à la conclusion que ce monde dynamique et transmodal ne correspond pas à une étape du développement de l'enfant, qui serait ensuite abandonnée pour laisser la place à d'autres modes de fonctionnement. Au contraire, cette strate d'expérience reste active tout au long de la vie, bien que généralement au-dessous du seuil de la

25. T. Reik, Écouter avec la troisième oreille: l'expérience intérieure d'un psychanalyste, Paris, Epi, 1976, p. 289.

26. Idem, p. 183.

27. Théétète 185a-186a.

28. De l'âme, II, 6, 418 a12 et 418 a18-20.

29. D. Stern, Le Monde interpersonnel du nourrisson, Paris, Presses universitaires de France, 1989. 


\section{L'attention}

conscience. Sous les perceptions, les émotions, les pensées et les actions qui constituent notre expérience consciente, cette strate silencieuse nous accompagne constamment, elle est l'étoffe même de notre expérience.

\section{Perméabilité des frontières}

Le plus souvent, la transmodalité de cette strate d'expérience s'accompagne d'une certaine perméabilité de la frontière habituellement perçue entre espace intérieur et espace extérieur. Nous faisons parfois cette expérience lorsque nous contemplons un tableau, qui soulève en nous un monde d'impressions fugitives d'intensité, de contraste, de résonance, ni objectives ni subjectives. Cette expérience se produit aussi à l'écoute d'une musique, ou même comme nous l'avons vu d'un simple son. Un tel sentiment de perméabilité des frontières entre mondes intérieur et extérieur, lié semble-t-il à une sorte d'accord rythmique entre les deux, semble se retrouver dans nos relations intersubjectives. Par exemple, il nous a été décrit par différents psychothérapeutes comme caractéristique de certains moments privilégiés de la cure thérapeutique.

\section{Transformation du sentiment d'identité}

Ce sentiment de dissolution des frontières est souvent associé à une transformation du sentiment d'identité individuelle : le sentiment d'être un « moi » solide et distinct du monde, devient plus « léger » et même disparaît. Par exemple, toutes les descriptions que j'ai recueillies de l'émergence d'une idée qu'on a coutume d'appeler une « intuition ${ }^{30}$ »- solution d'un problème, idée scientifique nouvelle, insight psychothérapeutique, intuition créatrice mentionnent un sentiment d'absence de contrôle: "Ça m'échappe ", "Ça $\mathrm{m}^{\prime}$ arrive », " Ça ne dépend pas de moi », "Ça m'est donné ». En cet instant, le sentiment dit "d'agentivité » (sense of agency en anglais), c'est-à-dire "le sentiment que c'est moi qui génère l'idée dans mon courant de conscience"31 est altéré, comme le confirme l'analyse des structures linguistiques utilisées pour décrire l'expérience. La forme active est en effet fréquemment remplacée par une forme plus passive : la personne décrivant l'expérience ne dit pas "j'ai une idée, je vois une image », mais "une idée me vient, une image $\mathrm{m}^{\prime}$ apparaît ${ }^{32}$ ». Il semble que le « sentiment d'appartenance » (sense of ownership), c'est-à-dire «le sentiment que cette idée est mon idée ${ }^{33}$ », soit aussi altéré, comme le suggère l'absence du pronom personnel «Je » dans

30. C. Petitmengin, L'expérience intuitive, Paris, L'Harmattan, 2001.

31. S. Gallagher (2000), "Philosophical conceptions of the self: implications for Cognitive Science », Trends in Cognitive Sciences, vol. 3, n 1, p. 14-21.

32. Rappelez-vous la célèbre conférence de Poincaré à l'Institut de psychologie de Paris : «Au moment où je mettais le pied sur le marchepied, l'idée me vint.» (H. Poincaré, Bulletin de l'Institut général de psychologie, $\mathrm{n}^{\circ} 3$, Paris, 1908).

33. S. Gallagher (2000), op. cit. 
beaucoup de descriptions. La personne décrivant l'expérience ne dit même plus " une idée $m e$ vient ", " une image $m^{\prime}$ apparaît », mais "il y a une idée, il y a une image ». L'expérience n'est pas ressentie comme étant la mienne, elle n'est pas vécue comme personnelle ${ }^{34}$.

Nous rencontrons ici un problème de vocabulaire. Car pour qualifier la dimension ressentie, où la distinction entre monde intérieur et monde extérieur, entre sujet et objet est absente, il devient difficile de parler d'expérience « subjective » ou « intérieure ».

\section{Micro-gestes pré-réfléchis d'accès à la dimension ressentie}

L'exploration de la dimension ressentie montre aussi que nous réalisons constamment de micro-gestes pour accéder à cette dimension, de manière ordinairement pré-réfléchie.

Par exemple, lorsque nous nous exprimons, nous réalisons d'instant en instant de micro-opérations, de micro-gestes pour entrer en contact avec le «sens ressenti » des idées que nous voulons exprimer. Ce processus a été particulièrement bien décrit par Gendlin ${ }^{35}$. Par exemple en ce moment, à chaque fois que je veux exprimer une idée, je dois retrouver le sens ressenti particulier qui sous-tend cette idée. Et si je n'ai pas de sens ressenti, je ne pourrai rien dire, ou alors des phrases vides de sens. Et si jamais je perds le «fil» de ce que je veux dire, si je perds le contact avec la dimension « ressentie », je ne pourrai pas continuer à écrire. Je chercherai à retrouver ce fil en dirigeant mon attention à l'intérieur de moi-même vers le sentiment de ce que je voulais dire, de telle sorte que les mots puissent jaillir à nouveau. De même, lorsque des mots me viennent pour exprimer cette idée, c'est en les confrontant avec ce sens ressenti, « ce quelque chose en moi qui n'est pas encore dit », que je sais si oui ou non ils sont justes. Par exemple, je cherche le mot « distiller ». J'en ai un sens intérieur, global, très difficile à décrire, et en même temps très précis car lorsqu'un mot au sens voisin me vient à l'esprit («fermenter»), je le rejette immédiatement. Non, «ce que je veux dire, et qui n'est pas encore dit » est plus précis. C'est une opération très

34. Cette expérience de perméabilité des frontières entre intérieur et extérieur et de dissolution $\mathrm{du}$ « Je », dont nous avons recueilli la description, ne semble pas trouver son équivalent dans la phénoménologie husserlienne. Pour Husserl, la continuité de l'ego est indispensable pour fonder la phénoménologie. Les analyses de P. Vermersch également font droit, au côté des pôles noétique et noématique, au pôle égoïque (par exemple dans «Conscience directe et conscience réfléchie", op. cit.). Alors que dans la tradition méditative vipashyana par exemple, la continuité de l'ego est une illusion, un peu comme celle d'un cercle décrit par un tison. La conscience et la subjectivité, le fait d'être un "soi", sont disjoints, le processus de prise de conscience est possible sans continuité, sans ego. La compréhension de cette divergence très profonde demanderait des développements qui dépasseraient le propos du présent article.

35. Par exemple E. Gendlin, «Introduction to thinking at the edge », ou « Making concepts from experience » (http://www.focusing.org). 


\section{L'attention}

subtile, ordinairement pré-réfléchie, de confrontation du mot avec le sens ressenti qui me permet de le savoir.

\section{Attention pré-réfléchie}

Ce qui semble particulièrement étonnant ici, c'est le caractère pré-réfléchi de ces micro-opérations. Des micro-gestes nous permettent, d'instant en instant, de prendre contact avec la dimension ressentie, en quelque sorte de tourner notre attention vers elle, tout en restant pré-réfléchis - puisqu'il faut un entraînement particulier pour prendre conscience de cette micro-activité. Il s'agit en quelque sorte d'une attention pré-réfléchie, ce qui paraît tout à fait paradoxal.

\section{Hypothèse de travail}

Revenons à la disposition attentionnelle qui nous permet, non de prendre un contact pré-réfléchi avec la dimension ressentie comme nous le faisons $\mathrm{d}$ 'instant en instant, mais d'en acquérir une conscience réfléchie. Cette disposition attentionnelle nous a été décrite comme le résultat d'un processus de défocalisation de l'attention et de relâchement de l'intentionnalité. L'interprétation de ces descriptions nous conduit à l'hypothèse suivante : ce processus pourrait correspondre à l'inversion, à la résorption en quelque sorte, d'un processus inverse de focalisation et de différenciation qui habituellement se reproduit d'instant en instant. La dimension ressentie correspondrait en réalité à une phase précoce d'une micro-genèse très rapide et pré-réfléchie, dont seules les phases les plus tardives apparaissent généralement à la conscience réfléchie. Cette phase précoce se caractérise par une absence de différenciation entre modalités sensorielles, entre espace intérieur et espace extérieur, entre sujet connaissant et objet connu. Elle est très rapidement suivie de gestes infimes d'identification, reconnaissance, catégorisation... qui aboutissent à l'identification et à l'appréciation d'un objet. Plus l'objet devient solide et stable, plus « mon » existence se confirme. La fixation de l'attention sur les phases tardives de ce processus occulte habituellement ses phases primitives. Seul le résultat du processus de différenciation apparaît à la conscience, sous la forme d'un monde et d'un «moi » cloisonnés. Mais le processus d'involution de l'intentionnalité précédemment décrit permet l'accès à la conscience réfléchie de phases de plus en plus précoces de cette micro-genèse. En d'autres termes, la dimension «ressentie» serait dernière dans la temporalité de la prise de conscience de l'expérience, mais première dans la temporalité de sa genèse.

Dans la mesure où notre démarche nous conduit ainsi à mettre entre parenthèses la distinction ordinairement perçue entre «intérieur» et « extérieur», entre «moi » et «monde», pour tenter de décrire finement le déploiement d'un phénomène, et notamment le processus de co-constitution 
de l'objectivité et de la subjectivité, nous considérons qu'elle relève d'une "phénoménologie génétique ${ }^{36}$ ».

L'affinement et la vérification de cette hypothèse de travail ${ }^{37}$ supposent l'adoption de méthodes adaptées. Par exemple, nous essayons actuellement de recueillir la description du déroulement micro-temporel de phénomènes mentaux très brefs ${ }^{38}$. Ce recueil est considérablement facilité lorsqu'il peut être fait auprès de pratiquants de vipashyanâ, puisque cette technique vise précisément à prendre conscience de la structure micro-dynamique de l'expérience. Une autre piste de vérification consiste à mettre au point des protocoles «neuro-expérientiels» adaptés. Non pas dans l'objectif de réduire les processus attentionnels à des processus neurophysiologiques, mais afin de créer un «cercle vertueux » d'enrichissement et d'affinement mutuel des analyses neurologiques et des analyses expérientielles. Par exemple, les trois dispositions attentionnelles détectées dans l'expérience auditive ont-elles des signatures neuronales spécifiques?

\section{Critique du terme " pré-réfléchi »}

L'exploration de la dimension « ressentie » nous conduit à douter de la pertinence du terme même "pré-réfléchi ", que nous continuons pour l'instant à employer faute d'en avoir trouvé un qui soit mieux adapté39. Pour plusieurs raisons. Tout d'abord, à cause du préfixe " pré » qui sous-entend que la conscience pré-réfléchie serait systématiquement suivie de conscience réfléchie.

Ensuite, à cause du terme « réfléchi » et de la métaphore du miroir qu'il introduit. Il semble problématique d'assimiler le processus de prise de conscience à un réfléchissement, et donc à un mouvement de prise de distance. Acquérir ce qu'on pourrait appeler la pleine conscience de son expérience ne semble pas consister à prendre du recul par rapport à elle pour l'observer, mais au contraire à s'en rapprocher, à entrer en contact avec elle.

La métaphore du réfléchissement induit aussi subrepticement l'idée qu'il y aurait entre conscience pré-réfléchie et conscience réfléchie une relation de

36. Projet décrit par Husserl dans De la synthèse passive (Grenoble, Millon, 1998).

37. Cette hypothèse est précisée dans C. Petitmengin, "L'énaction comme expérience vécue ", Intellectica, (2006), $\mathrm{n}^{\circ} 43$, p. 85-92, et dans C. Petitmengin, «Towards the source of thoughts. The gestural and transmodal dimension of lived experience », Journal of Consciousness Studies, vol. $14, \mathrm{n}^{\circ} 3$ (2007), p. 54-82.

38. L'objectif est proche de celui de la méthode de réalisation génétique (Aktualgenese), conçue par $\mathrm{H}$. Werner et ses successeurs dans le but d'obtenir la description des phases précoces d'une perception, ordinairement occultées par ses phases tardives (H. Werner, «Microgenesis and aphasia ", Journal of Abnormal Social Psychology, 52, 1956, p. 347-353).

39. Je me distancie sur ce point de On Becoming Aware, où la thématique du réfléchissement est très présente, la prise de conscience étant décrite comme acte réfléchissant. 


\section{L'attention}

correspondance, l'une copiant ou reflétant l'autre de manière plus ou moins exacte. Alors qu'au contraire au cours du processus de prise de conscience, une transformation subtile s'opère - fort heureusement car elle est la raison d'être même du processus - transformation qui reste à décrire finement ${ }^{40}$.

Enfin, et c'est le point le plus délicat, l'analyse des descriptions que nous avons recueillies suggère qu'au cours du processus de prise de conscience, plus l'attention relâche sa tension vers les objets extérieurs pour entrer en contact avec l'expérience dite «intérieure», plus la distinction entre « intérieur » et « extérieur », soi et non soi, se réduit.

Autrement dit, la pleine conscience ne semble pas s'accompagner de la conscience d'un soi. Dans la tradition phénoménologique, la conscience réfléchie l'est par et pour un sujet. Mais un pratiquant de vipashyanâ qui a passé des milliers d'heures à observer la micro-dynamique de son expérience, découvre que cette expérience, lorsqu'elle devient pleinement consciente d'elle-même au sens d'autoconsciente, réflexivement consciente, n'est pas l'expérience d'un sujet, d'un soi. Il ne trouve pas de soi, il n'en fait pas l'expérience. Comme le dit un sutra : "Quand le Tathâgata 41 entend l'audible, il ne crée pas un objet entendu et un sujet qui entend. Quand le Tathâgata voit le visible, il ne crée pas un objet vu et un sujet qui voit. Ainsi il n'est rien à quoi le Tathâgata s'accroche, il n'est rien qui le lie et qui le limite. $^{42}$ » Dans cette tradition, la conscience de soi n'est donc pas un trait constitutif de la pleine conscience.

Notre travail empirique rejoint donc sur ce point la tradition bouddhiste pour se distancier de la tradition phénoménologique. Pour cette dernière, l'expérience est toujours accompagnée d'une conscience de soi pré-réfléchie : l'expérience m'est toujours implicitement donnée comme mienne, elle a pour caractéristique intrinsèque d'être une expérience que je vis ${ }^{43}$, la conscience de l'objet perçu est toujours accompagnée de la conscience d'un sujet qui perçoit. L'absorption usuelle de l'attention dans l'objet a cependant pour effet de rendre cette conscience non remarquée ou pré-réfléchie, minimale : je suis conscient de moi-même de manière non thétique, seulement « du coin

40. Ces idées sont développées dans C. Petitmengin et M. Bitbol, « The Validity of First-Person Descriptions as Authenticity and Coherence ", Journal of Consciousness Studies 16 (2009), 11-12, p. 363-404.

41. Littéralement "celui qui est allé ainsi", celui qui a fait ce chemin (autrement dit un être pleinement éveillé, un « Bouddha »).

42. Kalakarama sutta, Anguttara-nikâya IV.24.

43. "If I am dizzy I can neither be in doubt nor mistaken about who the subject of my experience is, and it is nonsensical to ask whether I am sure that I am the one who is dizzy, or to demand a specification of the criteria being used by me in determining whether or not the felt dizziness is really mine. In effect, all of my experiences are characterized implicitly by a quality of mineness, that is, as having the quality of being experiences $I$ am undergoing or living through. (...) Pre-reflective self-consciousness is a constitutive feature and integral part of consciousness. » (Gallagher et D. Zahavi, The phenomenological mind, London, Routledge, 2008, p. 50). 
de l'œil » en quelque sorte, sans être réflexivement conscient de l'être, sans être conscient de cette conscience (de moi). Et cette conscience de soi préréfléchie est un trait constitutif de la conscience. Alors que dans la tradition bouddhiste, la conscience de soi est un leurre, créé précisément par l'absorption de l'attention dans l'objet perçu, qui entretient en retour l'illusion d'un sujet percevant. Nous croyons faire l'expérience d'un sujet au travers des objets, mais il s'agit d'une illusion - dont le statut a fait l'objet au cours des siècles de nombreux développements ${ }^{44}$. C'est le thème tout à fait fondateur et central du Bouddhisme: on ne fait jamais l'expérience d'un sujet, d'un soi. Ce qui n'est nullement incompatible avec la réflexivité de la conscience : pour les penseurs bouddhistes comme pour Husserl, Sartre et Merleau-Ponty, l'essence de la conscience est d'être consciente d'elle-même. Mais cette conscience autoconnaissante n'est pas la conscience d'un soi. De manière symétrique, l'intentionnalité n'est pas considérée comme constitutive de l'expérience. C'est ce que le méditant vérifie dans sa pratique : sujet et objet perdent progressivement leur solidité, perte d'intentionnalité et d'ipséité étant concomitantes. Cette pratique lui permet simultanément d'acquérir la "claire vision" (vipashyanâ) de la manière dont elles se co-constituent, compréhension dont l'épistémologie bouddhiste est une formalisation.

Dans la tradition phénoménologique, l'assimilation des deux types de réflexivité - conscience de soi et conscience d'un soi - explique probablement le glissement, particulièrement fréquent sous la plume de phénoménologues anglais, de l'expression "self-conscious ", à l'expression « conscious of a self ». Il nous semble cependant insuffisamment questionné.

C'est pour ces différentes raisons que le terme pré-réfléchi ne paraît pas bien adapté, et qu'il serait préférable d'en utiliser un autre. Pourquoi pas " non reconnu", qui se trouve d'ailleurs traduire le mot avidita utilisé en sanskrit pour qualifier cette caractéristique de l'expérience?

\section{Conclusion}

C'est dans la dimension ressentie de notre expérience, à la source de nos pensées, que semble se jouer notre rapport au monde. La reconnaissance de cette dimension n'est pas de l'ordre d'une compréhension intellectuelle, conceptuelle. Le mouvement d'apaisement de l'intentionnalité qu'elle requiert engage l'être tout entier. Dans ce processus, sujet, objet, et les points de repère qui nous permettent habituellement de structurer le monde perdent leur solidité, leur rigidité, deviennent de plus en plus légers. Mais étonnamment, lorsque nous renonçons à notre quête de points d'appui, se produit un grand apaisement. L'absence de frontières est absence d'interruption, de restriction. Elle permet au souffle, à la vie de circuler. Elle

44. Pour une introduction à cette question le lecteur peut se reporter à C. Petitmengin, Le Chemin du milieu. Introduction à la vacuité dans la pensée bouddhiste indienne, Paris, Dervy, 2007. 


\section{L'attention}

rend le mouvement, l'action plus fluides. Des solutions insoupçonnées apparaissent. Prendre contact avec la dimension profonde et spacieuse de notre expérience nous rassemble, nous réunifie, nous rend notre entièreté. Notre expérience redevient épaisse, pleine et riche de sens ${ }^{45}$. Dans cet espace non obstrué s'élève une sorte de douceur, de chaleur, de tendresse. Comme l'écrivait Francisco Varela en se référant à une très ancienne maxime bouddhiste, «la perte d'un point de référence fixe ou de fondements (...) est indissociable de la compassion, comme les deux côtés d'une médaille ou les deux ailes d'un oiseau 46 ». Retrouver le contact avec cette dimension pourrait être un enjeu crucial pour notre société.

Claire Petitmengin

Centre de recherche en épistémologie appliquée

(CREA - École Polytechnique/CNRS)

et Institut Télécom, Paris

45. "Nothing is as debilitating as a confused and distant functioning of experiencing. It is the chief malaise of our society » (E. Gendlin, Experiencing and the creation of meaning, p. 15-16) 46. Francisco Varela, Quel savoir pour l'éthique, Paris, La Découverte, 1996, p. 112. 